\title{
Existence and long-time behavior of solutions to the velocity-vorticity-Voigt model of the 3D Navier-Stokes equations with damping and memory
}

\author{
Nguyen $\operatorname{Toan}^{1}$ \\ ${ }^{1}$ Haiphong University
}

November 12, 2021

\begin{abstract}
In this paper, we study the long-time dynamical behavior of the non-autonomous velocity-vorticity-Voigt model of the 3D Navier-Stokes equations with damping and memory. We first investigate the existence and uniqueness of weak solutions to the initial boundary value problem for above-mentioned model. Next, we prove the existence of uniform attractor of this problem, where the time-dependent forcing term $\$ \mathrm{f} \backslash$ in $\mathrm{L}^{\wedge} 2 \_\mathrm{b}\left(\backslash \operatorname{mathbb}\{\mathrm{R}\} ; \mathrm{H}^{\wedge}\{-1\}(\backslash\right.$ Omega $\left.)\right) \$$ is only translation bounded instead of translation compact. The results in this paper will extend and improve some results in Yue, Wang (Comput. Math. Appl., 2020) in the case of non-autonomous and contain memory kernels which have not been studied before.
\end{abstract}

\section{Hosted file}

Toan_6.11.21.pdf available at https://authorea.com/users/445763/articles/545173-existenceand-long-time-behavior-of-solutions-to-the-velocity-vorticity-voigt-model-of-the-3dnavier-stokes-equations-with-damping-and-memory 